\title{
Efficient open domination in graph products
}

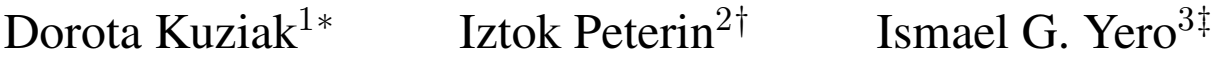 \\ ${ }^{1}$ Departament d'Enginyeria Informàtica i Matemàtiques, Universitat Rovira i Virgili, Spain \\ ${ }^{2}$ Faculty of Electrical Engineering and Computer Science, University of Maribor, Slovenia \\ ${ }^{3}$ Departamento de Matemáticas, EPS Algeciras, Universidad de Cádiz, Spain \\ received $9^{\text {th }}$ Oct. 2013, revised 22 ${ }^{\text {nd }}$ Jan. 2014, 14 $4^{\text {th }} \mathrm{Feb} .2014$, accepted $24^{\text {th }} \mathrm{Feb} .2014$.
}

A graph $G$ is an efficient open domination graph if there exists a subset $D$ of $V(G)$ for which the open neighborhoods centered in vertices of $D$ form a partition of $V(G)$. We completely describe efficient open domination graphs among lexicographic, strong, and disjunctive products of graphs. For the Cartesian product we give a characterization when one factor is $K_{2}$.

Keywords: efficient open domination; graph products; total domination

\section{Introduction and preliminaries}

Let $G$ be a graph with vertex set $V(G)$ and edge set $E(G)$. We use standard notations $N_{G}(v)$ for the open neighborhood $\{u: u v \in E(G)\}$ and $N_{G}[v]$ for the closed neighborhood $N_{G}(v) \cup\{v\}$ for a graph $G$. By $u \sim v$ we denote adjacency of $u$ and $v$ from $V(G)$. Throughout the article we consider only simple graphs.

The domination number $\gamma(G)$ of a graph $G$ is a classical invariant in graph theory. It is the minimum cardinality of a set $S$ for which the union of closed neighborhoods centered in vertices of $S$ cover the whole vertex set of $G$. Such a set $S$ is called a dominating set of $G$. Hence each vertex of $G$ is either in $S$ or adjacent to a vertex in $S$. In other words, we can say that vertices of $S$ control each vertex outside of $S$. A classical question in such a situation is: who controls the vertices of $S$ ? One possible solution to this dilemma is the total domination. A set $D \subseteq V(G)$ is a total dominating set of $G$ if every vertex of $G$ is adjacent to a vertex of $D$. (Hence, also vertices of $D$ are controlled by $D$.) The total domination number of a graph $G$ is the minimum cardinality of a total dominating set of $G$ and is denoted by $\gamma_{t}(G)$. A total dominating set $D$ of cardinality $\gamma_{t}(G)$ is called $\gamma_{t}(G)$-set.

The natural question for a graph $G$ is whether we can find a total dominating set $D$ for which its open neighborhoods not only cover $V(G)$ but also form a partition of $V(G)$, which means that $N_{G}(u) \cap$

\footnotetext{
*Email: dorota.kuziak@urv.cat.

${ }^{\dagger}$ Email: iztok.peterin@um. si. The author is also with IMFM, Slovenia.

$\ddagger_{E}$ Email: ismael.gonzalez@uca.es. The research was done during the visit of the University of Maribor, Slovenia, supported by "Ministerio de Educación, Cultura y Deporte", Spain, under the "Jose Castillejo" program for young researchers. Reference number: CAS12/00267.
}

1365-8050 @ 2014 Discrete Mathematics and Theoretical Computer Science (DMTCS), Nancy, France 
$N_{G}(v)=\emptyset$ for every different $u, v \in D$. The problem has been presented under the names: total perfect codes [3], efficient open domination [7] and exact transversals [4]. In the present work we follow the terminology of efficient open domination. A graph $G$ is an efficient open domination graph if there exists a set $D$, called an efficient open dominating set, for which $\bigcup_{v \in D} N_{G}(v)=V(G)$ and $N_{G}(u) \cap N_{G}(v)=\emptyset$ for every pair $u$ and $v$ of distinct vertices of $D$. It is easy to see that paths $P_{n}$ are efficient open domination graphs if and only if $n \not \equiv 1(\bmod 4)$, while cycles $C_{n}$ are efficient open domination graphs if and only if $n \equiv 0(\bmod 4)$.

The problem of establishing whether a graph $G$ is an efficient open domination graph is an NPcomplete problem, see [16] (or [8]). This issue has been studied under its different names, nevertheless it has received not enough attention. Efficient open domination trees have been characterized recursively in [8]. Various properties of efficient open domination graphs were presented in [7]. The efficient open domination graphs among Cayley graphs were studied in [20] and the efficient open domination grid graphs in [4, 5, 14]. Moreover, the efficient open domination graphs among direct product graphs were characterized in [1]. However we have not found the following basic connection in the literature.

Observation 1.1 If $G$ is an efficient open domination graph with an efficient open dominating set $D$, then $\gamma_{t}(G)=|D|$.

Proof. If $D$ is an efficient open dominating set of $G$, then $D$ is also a total dominating set of $G$ and $\gamma_{t}(G) \leq|D|$ follows. On the other hand, an arbitrary vertex of $D$ has at least one neighbor in every $\gamma_{t}(G)$ set $D^{\prime}$, since $\bigcup_{v \in D^{\prime}} N_{G}(v)=V(G)$. Moreover, these neighbors must be different, since $\bigcup_{v \in D} N_{G}(v)$ form a partition of $V(G)$. Hence $\gamma_{t}(G) \geq|D|$ and the equality follows.

From this observation immediately follows that all efficient open dominating sets of a graph $G$ have the same cardinality. Another simple fact holds for regular graphs, since vertices from an efficient open dominating set are given by disjoint pairs of adjacent vertices and all the vertices have the same number of neighbors.

Observation 1.2 Let $G$ be an $r$-regular graph of order $n$. If $G$ is an efficient open domination graph, then $n \equiv 0(\bmod (2 r))$.

A similar approach is also known for dominating sets under the name 1-perfect graphs. That is, $G$ is 1-perfect graph if there exists a set $P \subseteq V(G)$ for which $V(G)=\bigcup_{v \in P} N_{G}[v]$ and $N_{G}[u] \cap N_{G}[v]=\emptyset$ for every pair $u$ and $v$ of distinct vertices of $P$. Set $P$ is called a perfect code of $G$. The name arose from codes, since one can discover and repair one error in such graphs.

Several graph products have been investigated in the last few decades and a rich theory involving the structure and recognition of classes of these graphs has emerged, cf. [10]. The most studied graph products are the Cartesian product, the strong product, the direct product and the lexicographic product which are also called standard products. One standard approach to graph products is to deduce properties of a product with respect to (the same) properties of its factors. See a short collection of these types involving total domination and perfect codes in [6, 9, 11, 12, 13, 15, 17].

The domination related problems on the Cartesian product seems to be the most problematic among standard products. We just mention the famous Vizing's conjecture: $\gamma(G \square H) \geq \gamma(G) \gamma(H)$, which is probably the most challenging problem in the area of domination (see the latest survey on Vizing's conjecture [2]). The efficient open domination is no exception. 
In the next section we completely describe the efficient open domination graphs among strong, lexicographic, and disjunctive products. After that we introduce zig-zag graphs and explore their structure. They are used in the following section where we completely describe graphs for which their Cartesian product with $K_{2}$ is an efficient open domination graph. We end with a section on grid-like graphs.

\section{The lexicographic, strong, and disjunctive products}

The lexicographic product $G \circ H$ (sometimes also denoted by $G[H]$ ) of graphs $G$ and $H$ is a graph with $V(G \circ H)=V(G) \times V(H)$. Two vertices $(g, h)$ and $\left(g^{\prime}, h^{\prime}\right)$ are adjacent in $G \circ H$ whenever $g g^{\prime} \in E(G)$ or $\left(g=g^{\prime}\right.$ and $\left.h h^{\prime} \in E(H)\right)$. For a fixed $h \in V(H)$ we call $G^{h}=\{(g, h) \in V(G \circ H): g \in V(G)\}$ a $G$-layer in $G \circ H$. Symmetrically $H$-layers ${ }^{g} H$ for a fixed $g \in V(G)$ are defined. Notice that the subgraph of $G \circ H$ induced by a $G$-layer or an $H$-layer is isomorphic to $G$ or $H$, respectively. The map $p_{G}: V(G \circ H) \rightarrow V(G)$ defined by $p_{G}((g, h))=g$ is called a projection map onto $G$. Similarly, we define $p_{H}$ as the projection map onto $H$. Projections are defined as maps between vertices, but frequently it is more comfortably to see them as maps between graphs. In this case we observe the subgraphs induced by $A \subseteq V(G \circ H)$ and $p_{X}(A)$ for $X \in\{G, H\}$. The lexicographic product is clearly not commutative, nevertheless it is associative [10].

Theorem 2.1 Let $G$ and $H$ be graphs. The lexicographic product $G \circ H$ is an efficient open domination graph if and only if either

(i) $G$ is a graph without edges and $H$ is an efficient open domination graph, or

(ii) $G$ is an efficient open domination graph and $H$ contains an isolated vertex.

Proof. If $G$ is a graph without edges on $n$ vertices, then $G \circ H$ is isomorphic to $n$ copies of $H$. If in addition $H$ is an efficient open domination graph, then also $n$ copies of $H$ form an efficient open domination graph. Now, let $G$ be an efficient open domination graph, let $D_{G}$ be one efficient open dominating set and let $h_{0}$ be an isolated vertex of $H$. We will show that $D_{G} \times\left\{h_{0}\right\}$ is an efficient open dominating set of $G \circ H$. For this, notice that $N_{G \circ H}\left(g, h_{0}\right)=N_{G}(g) \times V(H)$ and $\bigcup_{g \in D_{G}} N_{G \circ H}\left(g, h_{0}\right)=V(G \times H)$. If $g, g^{\prime} \in D_{G}$ and $g \neq g^{\prime}$, then $N_{G \circ H}\left(g, h_{0}\right) \cap N_{G \circ H}\left(g^{\prime}, h_{0}\right) \neq \emptyset$ implies that $N_{G}(g) \cap N_{G}\left(g^{\prime}\right) \neq \emptyset$, which is a contradiction. Therefore, $G \circ H$ is an efficient open domination graph.

Conversely, let $G \circ H$ be an efficient open domination graph with an efficient open dominating set $D$. Let $(g, h),\left(g^{\prime}, h^{\prime}\right) \in D$ be adjacent vertices. Suppose first that there exists such an edge with $g \neq g^{\prime}$. If $h^{\prime \prime} \in N_{H}(h)$, then $\left(g, h^{\prime \prime}\right) \in N_{G \circ H}(g, h) \cap N_{G \circ H}\left(g^{\prime}, h^{\prime}\right)$, which is a contradiction. Hence $h$ (and by symmetry also $h^{\prime}$ ) is an isolated vertex of $H$. Since $H$ contains an isolated vertex, it follows that $G$ has no isolated vertices, otherwise $G \circ H$ would contain isolated vertices, which is impossible for an efficient open domination graph. Thus, the fact $\left(g_{1}, h_{1}\right),\left(g_{2}, h_{2}\right) \in D$, implies that $h_{1}$ and $h_{2}$ are isolated vertices of $H$ (notice that it can happen $h_{1}=h_{2}$ ). If $g_{1} \in N_{G}(g) \cap N_{G}\left(g^{\prime}\right)$ for some $g, g^{\prime} \in$ $p_{G}(D)$, then ${ }^{g_{1}} H \subseteq N_{G \circ H}(g, h) \cap N_{G \circ H}\left(g^{\prime}, h^{\prime}\right)$ for $(g, h),\left(g^{\prime}, h^{\prime}\right) \in D$, which is a contradiction. Also $\bigcup_{g_{1} \in p_{G}(D)} N_{G}\left(g_{1}\right)=V(G)$, since $\bigcup_{\left(g_{1}, h_{1}\right) \in D} N_{G \circ H}\left(g_{1}, h_{1}\right)=V(G \circ H)$ and $D$ is an efficient open dominating set. Thus, $G$ is an efficient open domination graph (with an efficient open dominating set $\left.p_{G}(D)\right)$.

Now we can assume that all edges between vertices of $D$ have the same first coordinate: $(g, h)\left(g, h^{\prime}\right)$. Thus, $g$ is an isolated vertex of $G$, otherwise ${ }^{g^{\prime}} H \subseteq N_{G \circ H}(g, h) \cap N_{G \circ H}\left(g, h^{\prime}\right)$ for any neighbor $g^{\prime}$ 
of $g$ in $G$, which is not possible. Since $\left\{N_{G \circ H}(g, h):(g, h) \in D\right\}$ forms a partition of $G \circ H$, every vertex $\left(g_{1}, h_{1}\right)$ is in some $N_{G \circ H}(g, h)$. Again $(g, h)$ is in some $N_{G \circ H}\left(g^{\prime}, h^{\prime}\right)$ and we have $g=g^{\prime}=g_{1}$. Hence every vertex of $G$ is an isolated vertex and suppose that there are $n$ vertices in $G$. Every $H$-layer is isomorphic to $H$ and $G \circ H$ is isomorphic to $n$ copies of $H$. Since $G \circ H$ is an efficient open domination graph, every component of $G \circ H$ is such. Therefore, also $H$ is an efficient open domination graph which ends the proof.

We can easily generalize the construction of Theorem 2.1 (ii). Namely, let $G$ be any efficient open domination graph with $V(G)=\left\{g_{1}, \ldots, g_{n}\right\}$. Choose $n$ arbitrary graphs $H_{1}, \ldots, H_{n}$. Let $N_{G}\left(g_{i}\right)=$ $\left\{g_{j_{1}}, g_{j_{2}}, \ldots, g_{j_{k_{i}}}\right\}$ for every $i \in\{1, \ldots, n\}$. Connect $g_{i}$ by an edge with every vertex of graphs $H_{j_{1}}, H_{j_{2}}, \ldots, H_{j_{k_{i}}}$ to obtain a new graph $G^{*}$. It is easy to see that an efficient open dominating set $D$ of $G$ is also an efficient open dominating set of $G^{*}$. Moreover, we can add to $G^{*}$ arbitrary many edges between vertices of $H_{i}$ and $H_{j}$ as long as $g_{i} g_{j} \in E(G)$ and the obtained graph is still an efficient open domination graph. If we add all possible edges between $H_{i}$ and $H_{j}$ whenever $g_{i} g_{j} \in E(G)$ and all graphs $H_{1}, \ldots, H_{n}$ are isomorphic to a graph $H$, then the new graph is isomorphic to $G \circ(H \cup\{h\})$ where $h$ is an isolated vertex of $H \cup\{h\}$.

The strong product $G \otimes H$ of graphs $G$ and $H$ is a graph with $V(G \otimes H)=V(G) \times V(H)$. Two vertices $(g, h)$ and $\left(g^{\prime}, h^{\prime}\right)$ are adjacent in $G \otimes H$ whenever $\left(g g^{\prime} \in E(G)\right.$ and $\left.h=h^{\prime}\right)$ or $\left(g=g^{\prime}\right.$ and $\left.h h^{\prime} \in E(H)\right)$ or $\left(g g^{\prime} \in E(G)\right.$ and $\left.h h^{\prime} \in E(H)\right)$. The commutativity of the strong product follows from the symmetry of the definition of adjacency and for associativity see [10].

Since $G \circ H \cong G \otimes H$ if $G$ is a graph without edges, Theorem 2.1 (i) already gives a hint for the strong product. Surprisingly, these are the only graphs (up to the commutativity of the factors) among strong products which are efficient open domination graphs. This follows immediately from the fact that $\left|N_{G \otimes H}(g, h) \cap N_{G \bowtie H}\left(g^{\prime}, h^{\prime}\right)\right| \geq 2$ for any two adjacent vertices $(g, h)$ and $\left(g^{\prime}, h^{\prime}\right)$, where both $g$ and $h$ are not isolated vertices of $G$ and $H$, respectively.

Proposition 2.2 Let $G$ and $H$ be two graphs. The strong product $G \otimes H$ is an efficient open domination graph if and only if one factor is a graph without edges and the other is an efficient open domination graphs.

The disjunctive product $G \oplus H$ of graphs $G$ and $H$ is a graph with $V(G \oplus H)=V(G) \times V(H)$. Two vertices $(g, h)$ and $\left(g^{\prime}, h^{\prime}\right)$ are adjacent in $G \oplus H$ whenever $g g^{\prime} \in E(G)$ or $h h^{\prime} \in E(H)$. Like the Cartesian and the direct product, the disjunctive product is also commutative. This notion of graph product was introduced by Ore [18], where it was called the Cartesian sum of graphs. The name disjunctive product is due to [19].

Theorem 2.1 (ii) gives a hint for a characterization of efficient open domination graphs, for the case of disjunctive product, which has quite similar behavior like lexicographic product. Thus, we have the following result, which proof is quite similar to the proof of Theorem 2.1

Theorem 2.3 The disjunctive product of two graphs is an efficient open domination graph if and only if one graph is an efficient open domination graph and the other one contains an isolated vertex.

Proof. Let $G$ and $H$ be any graphs. Assume that $G$ is an efficient open domination graph with an efficient open dominating set $D_{G}$ and let $h_{0}$ be an isolated vertex of $H$. Proceeding like in the proof of Theorem 
2.1 we show that $D_{G} \times\left\{h_{0}\right\}$ is an efficient open dominating set of $G \oplus H$. Therefore, $G \oplus H$ is an efficient open domination graph.

On the contrary, we consider $G \oplus H$ is an efficient open domination graph with an efficient open dominating set $D$ and we also proceed as in the proof of Theorem 2.1 Let $(g, h),\left(g^{\prime}, h^{\prime}\right) \in D$ be adjacent vertices. If $g \neq g^{\prime}$, then $h$ (and by symmetry also $h^{\prime}$ ) is an isolated vertex of $H$. Since $H$ contains an isolated vertex, it follows that $G$ has no isolated vertices, otherwise $G \oplus H$ would contain isolated vertices, which is impossible for an efficient open domination graph. Thus, if $\left(g_{1}, h_{1}\right),\left(g_{2}, h_{2}\right) \in$ $D$, then $h_{1}$ and $h_{2}$ are isolated vertices of $H$. If $g_{1} \in N_{G}(g) \cap N_{G}\left(g^{\prime}\right)$ for some $g, g^{\prime} \in p_{G}(D)$, then ${ }^{g_{1}} H \subseteq N_{G \oplus H}(g, h) \cap N_{G \oplus H}\left(g^{\prime}, h^{\prime}\right)$ for $(g, h),\left(g^{\prime}, h^{\prime}\right) \in D$, which is a contradiction. Also $\bigcup_{g_{1} \in p_{G}(D)} N_{G}\left(g_{1}\right)=V(G)$, since $\bigcup_{\left(g_{1}, h_{1}\right) \in D} N_{G \oplus H}\left(g_{1}, h_{1}\right)=V(G \oplus H)$ and $D$ is an efficient open dominating set. Thus, $G$ is an efficient open domination graph (with an efficient open dominating set $\left.p_{G}(D)\right)$.

Similarly, if $h \neq h^{\prime}$, then we obtain that $g_{1}$ and $g_{2}$ are isolated vertices of $G$. An analogous procedure shows that, in this case, $H$ is an efficient open domination graph. Therefore, the proof is complete.

For the case of direct product graphs, the efficient open domination graphs among them were already characterized in [1]. By completeness we include their result in this section. The direct product $G \times H$ of graphs $G$ and $H$ is a graph with vertex set $V(G \times H)=V(G) \times V(H)$. Two vertices $(g, h)$ and $\left(g^{\prime}, h^{\prime}\right)$ are adjacent in $G \times H$ whenever $g g^{\prime} \in E(G)$ and $h h^{\prime} \in E(H)$. The open neighborhoods of vertices in direct product graphs are nicely connected to open neighborhoods of projections to the factors. Namely, $N_{G \times H}(g, h)=N_{G}(g) \times N_{H}(h)$ for every vertex $(g, h) \in V(G \times H)$ and this is the main reason for nice behavior of efficient open domination graphs among direct product with respect to its factors.

Theorem 2.4 [1] Let $G$ and $H$ be two graphs. The direct product $G \times H$ is an efficient open domination graph if and only if $G$ and $H$ are efficient open domination graphs.

By Observation, 1.1 the following corollary is obtained.

Corollary 2.5 If $G$ and $H$ are efficient open domination graphs, then $\gamma_{t}(G \times H)=\gamma_{t}(G) \gamma_{t}(H)$.

\section{The zig-zag graphs}

This section deals with a family of graphs which is the heart of the characterization of the efficient open domination graphs among the so called prism graphs $G \square K_{2}$. In this sense, we give some deep description of such a family. To do so, we need to introduce some notation. By $d_{G}(u, v)$ we mean the geodesic or shortest path distance, which is the number of edges on a shortest $u, v$-path in $G$. Distance $d_{G}(e, v)$ between edge $e$ and a vertex $v$ in $G$ is the shortest distance between end vertices of $e$ and $v$, while the distance $d_{G}\left(e_{1}, e_{2}\right)$ between edges $e_{1}$ and $e_{2}$ is the shortest distance between end vertices of $e_{1}$ and end vertices of $e_{2}$. In general, for $P, Q \subseteq V(G)$, the distance $d_{G}(P, Q)$ between them is the shortest distance between a vertex from $P$ and a vertex from $Q$. Two different edges are incident if they have a common end vertex.

Let $G$ be a graph on at least three vertices and $E^{\prime}=\left\{e_{1}, \ldots, e_{k}\right\}$ be a subset of $E(G)$, where $e_{i}=u_{i} v_{i}$, with the following properties:

(i) $N_{G}\left(u_{i}\right) \cap N_{G}\left(v_{i}\right)=\emptyset$; 
(ii) $d_{G}\left(e_{i}, e_{j}\right) \geq 2$ for every different pair $i, j \in\{1, \ldots, k\}$;

(iii) for every $w \in V(G)-\left\{u_{i}, v_{i}: i \in\{1, \ldots, k\}\right\}$ there exist unique $j$ and $\ell, j \neq \ell$, such that $d\left(w, e_{j}\right)=d\left(w, e_{\ell}\right)=1$

(iv) for every sequence of distinct edges $e_{i_{1}}, e_{i_{2}}, \ldots, e_{i_{j}}, j>2$, with $d_{G}\left(e_{i_{\ell}}, e_{i_{\ell+1(\bmod j)}}\right)=2$ for $\ell \in\{1, \ldots, j\}, j$ must be an even number.

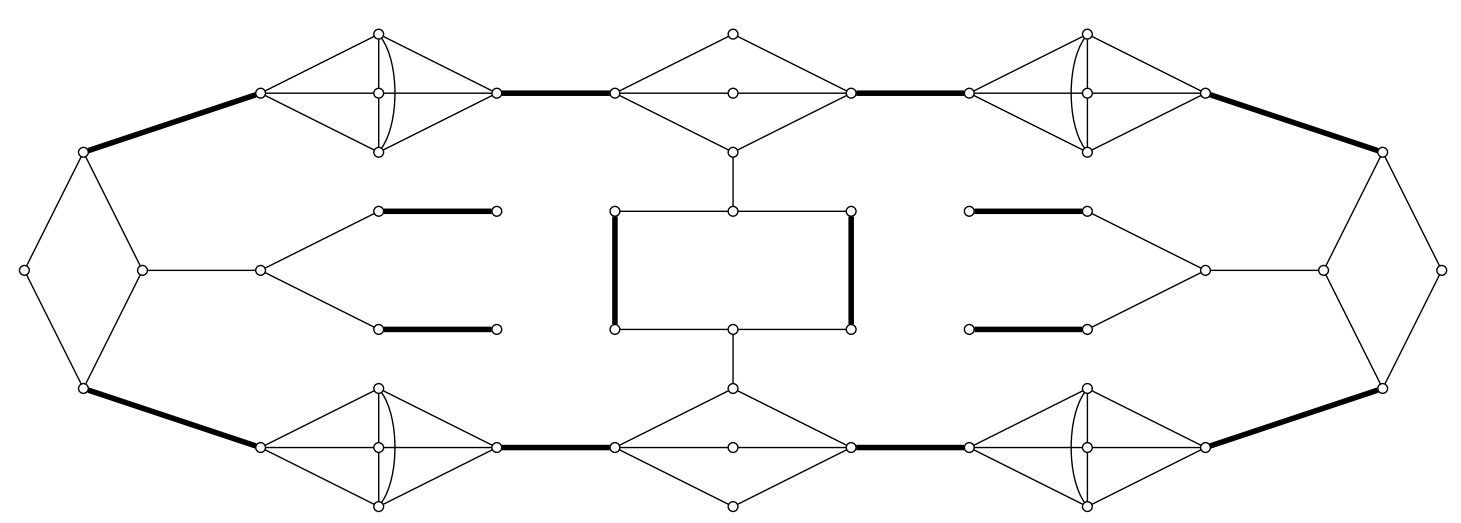

Fig. 1: A zig-zag graph where the zig-zag set is formed by the edges in bold.

We call $E^{\prime}$ a zig-zag set of $G$ and, if there exists a zig-zag set in $G$, we call $G$ as a zig-zag graph. (The motivation for this name follows from the property (iv) and the zig-zag role of edges at distance 2 in efficient open domination set of $G \square K_{2}$ as can be seen in the following section.) In Figure 1 appears an example of a zig-zag graph, where the zig-zag set is given by the edges in bold. Notice that zig-zag graphs among cycles are exactly $C_{6 k}$ for a positive integer $k$ (we need six to fulfill the property (iv) of the definition). Also, if a zig-zag graph $G$ contains a vertex $v$ of degree one, then the edge $v u \in E(G)$ must be in any zig-zag set of $G$. This follows from property (iii), since any vertex of degree one has not two different non incident edges at distance one. Thus, for instance, a path $P_{n}$ is a zig-zag graph whenever $n=2+3 k$ for some positive integer $k$. Moreover, observe that if a zig-zag graph $G$ contains no cycles, then property (iv) is fulfilled. Hence, we need a cycle $C$ for property (iv) to be nontrivial. If edges from a zig-zag set $E^{\prime}$ which have at least one end vertex on $C$ are successively at distance two, then its number must be even. If this holds for all such cycles of a graph $G$, then property (iv) is fulfilled for $G$.

Since zig-zag graphs play an important role in the efficient open domination graphs among prisms, we give somewhat deeper insight into their structure. Next we consider a construction that describes the family $\mathcal{G}$ of all graphs that satisfy properties (i), (ii), and (iii) of the definition. We begin with an arbitrary graph $H$ on $n$ vertices, and let $E^{\prime}=\left\{e_{i}: e_{i}=u_{i} v_{i}, i \in\{1, \ldots, r\}\right\}$ be a set of $r$ disjoint pairs of edges, with $r \geq 2$. Now, to obtain a graph $G \in \mathcal{G}$, for every vertex $h \in V(H)$ choose two edges $e_{j}, e_{\ell} \in E^{\prime}$ and connect $h$ by an edge with exactly one end vertex of $e_{j}$ and one of $e_{\ell}$. Notice that we can choose the same edges for different vertices. It is straightforward to observe that $G$ is a graph satisfying properties (i), (ii), and (iii) for the set $E^{\prime}$.

On the other hand, if $G$ is a graph carrying out the properties (i), (ii), and (iii) for the set of edges $F \subseteq E(G)$, then $F$ represents $E^{\prime}$ and the subgraph induced by $V(G)-V(F)$ represents $H$ in the above 
description. Thus, this construction gives a characterization of all graphs for which properties (i), (ii), and (iii) hold. However, property (iv) does not always hold in this construction.

We will incorporate property (iv) to the above construction in two special cases, which also reveals richness of the structure of zig-zag graphs. The first case is related to the following. If $G \in \mathcal{G}$ was constructed in such a way that every $v \in V(G)$ has two private edges at distance one, then such edges do not lie in any cycle, and, as mentioned before, property (iv) is fulfilled. As a consequence, $G$ is a zig-zag graph. Notice that in this construction we need $2 n$ disjoint edges in $E^{\prime}$, where $n$ is the order of the graph $H$ used to generate $G$.

For the second construction of zig-zag graphs, we do the following. Let $H$ be an arbitrary graph of order $n$ and let $E=\left\{e_{i}: e_{i}=u_{i} v_{i}, i \in\{1, \ldots, r\}\right\}$ be a set of disjoint edges not in $H$ where $r=n$ if $n$ is even, or $r=n+1$ if $n$ is odd. We generate a zig-zag graph $G$ as follows.

- We partition the set $V(H)$ into the sets $S_{1}, \ldots, S_{t}$ such that at most one set, say $S_{t}$, has cardinality one and other ones have even cardinality.

- We partition the set $E$ into the sets $E_{1}, \ldots, E_{t}$ such that, $\left|E_{i}\right|=\left|S_{i}\right|$ for every $i \in\{1, \ldots, t-1\}$ and, if $n$ is odd, then $\left|E_{t}\right|=2$ or, if $n$ is even, then $\left|E_{t}\right|=\left|S_{t}\right|$.

- Let $S_{i}=\left\{x_{i 1}, x_{i 2}, \ldots, x_{i\left|S_{i}\right|}\right\}$ and let $E_{i}=\left\{e_{i_{1}}, e_{i_{2}}, \ldots, e_{i_{\left|S_{i}\right|}}\right\}$.

- Now, for every $i \in\{1, \ldots, t-1\}$, we add edges between $S_{i}$ and the vertices of $E_{i}$ in such a way that we form the cycle $x_{i 1} u_{i_{1}} v_{i_{1}} x_{i 2} u_{i_{2}} v_{i_{2}} \ldots x_{i\left|S_{i}\right|} u_{i_{\left|S_{i}\right|}} v_{i_{\left|S_{i}\right|}} x_{i 1}$.

- Now, if $n$ is even, then we proceed with $S_{t}$ as above. On the contrary, if $n$ is odd, then $S_{t}=\left\{x_{t 1}\right\}$ and $E_{t}=\left\{e_{t_{1}}, e_{t_{2}}\right\}$. Hence, we form the path $u_{t_{1}} v_{t_{1}} x_{t 1} u_{t_{2}} v_{t_{2}}$.

Notice that a graph $G$ obtained by such a construction is a zig-zag graph with zig-zag set $E$. It is also easy to see that there are more zig-zag graphs (with less edges in zig-zag set). Take for instance, the complete graph $K_{2 n}$ and choose for a set $E^{\prime}$ to be any perfect matching of $K_{2 n}$. In addition subdivide by one vertex every remaining edge of $K_{2 n}$. It is straightforward to observer that the obtained graph, $K_{2 n}^{+}$is a zig-zag graph with zig-zag set $E^{\prime}$.

We end the discussion on zig-zag graphs by a recursive description of the family $\mathcal{T}$ of all zig-zag trees. Since trees have no cycles, it follows that any tree $T$ is a zig-zag graph if and only it satisfies properties (i), (ii), and (iii). Recall that a path $P_{n}$ is a zig-zag graph whenever $n=2+3 k$ for some positive integer $k$. Let $T_{1}$ and $T_{2}$ be zig-zag trees with corresponding zig-zag sets $E_{1}^{\prime}$ and $E_{2}^{\prime}$, respectively. The following two rules can be used to obtain a tree $T$ by a zig-zag tree construction.

(a) choose $e_{1}=u_{1} v_{1} \in E_{1}^{\prime}$ and $e_{2}=u_{2} v_{2} \in E_{2}^{\prime}$ and then identify the vertices $u_{1}$ with $u_{2}$ (hence vertex $u$ ) and $v_{1}$ with $v_{2}$ (hence vertex $v$ ) to maintainin the edge $u v$ in $T$;

(b) choose any $x \in V\left(T_{1}\right)-V\left(E_{1}^{\prime}\right)$ and any $y \in V\left(T_{2}\right)-V\left(E_{2}^{\prime}\right)$ and connect $x$ with $y$ by an edge to obtain $T$.

We say that a tree $T \in \mathcal{T}$ if it is obtained from zig-zag trees $T_{1}$ and $T_{2}$ by one of the above rules (a zig-zag tree construction) or $T$ is $P_{5}$.

Theorem 3.1 A tree $T$ is a zig-zag graph if and only if $T \in \mathcal{T}$. 
Proof. If $T$ is isomorphic to $P_{5}$, then it is clearly a zig-zag tree. So, suppose that $T \in \mathcal{T}$ obtained from zig-zag trees $T_{1}$ and $T_{2}$ with corresponding zig-zag sets $E_{1}^{\prime}$ and $E_{2}^{\prime}$, respectively. Let $E^{\prime}=E_{1}^{\prime} \cup E_{2}^{\prime}$. Notice that properties (i),(ii), and (iii) of the definition of zig-zag set are clearly satisfied for $E^{\prime}$ in $T$ after applying rule (a) or (b) to $T_{1}$ and $T_{2}$, since $E_{1}^{\prime}$ and $E_{2}^{\prime}$ are zig-zag sets. Since trees have no cycles, we do not need to check property (iv). Therefore, $T$ is a zig-zag tree.

Conversely, let $T$ be a zig-zag tree with corresponding zig-zag set $E^{\prime}$. If $T$ is isomorphic to $P_{5}$, then $T \in \mathcal{T}$. If $T$ is isomorphic to $P_{2+3 k}$ for $k \geq 2$, then it can be obtained from $P_{5}$ and $P_{2+3(k-1)}$ by using rule (a) of the zig-zag tree construction. Therefore, $T \in \mathcal{T}$.

Assume now that there exists a vertex $v$ of degree at least three in $T$. If $v$ is not an end vertex of any edge of $E^{\prime}$, then exactly two vertices $x, y \in N(v)$ are end vertices of an edge in $E^{\prime}$ by property (iii). Choose any neighbor $u \in N(v)-\{x, y\}$. By property (iii) there exists two edges $e_{u}, e_{u}^{\prime} \in E^{\prime}$ that are at distance one to $u$. Now, delete edge $u v$ from $T$. Clearly $T$ splits into two trees $T_{u}$ and $T_{v}$, where $T_{u}$ contains $u$ and $T_{v}$ contains $v$. Consider the sets of edges $E_{u}=E^{\prime} \cap E\left(T_{u}\right)$ and $E_{v}=E^{\prime} \cap E\left(T_{v}\right)$. Notice that properties (i) and (ii) hold for $E_{u}$ and $E_{v}$, since $E^{\prime}$ is a zig-zag set. Property (iii) must be checked only for $u$. But both $e_{u}$ and $e_{u}^{\prime}$ must be in $E_{u}$, since $v$ is not an end vertex of any edge of $E^{\prime}$. So, property (iii) is clear for $E_{u}$ and $E_{v}$ in $T_{u}$ and $T_{v}$, respectively. Thus, $T_{u}$ and $T_{v}$ are zig-zag graphs with zig-zag sets $E_{u}$ and $E_{v}$, respectively. Moreover, $T$ is obtained from $T_{u}$ and $T_{v}$ by using rule (b). Therefore, $T \in \mathcal{T}$.

Now let $v$ be an end vertex of the edge $e \in E^{\prime}$ and let $e=u v$. We shall "divide" the tree $T$ using a kind of "reverse-like process" of rule (a) into two trees $T_{u}$ and $T_{v}$, where $T_{u}$ contains $u$ and $T_{v}$ contains $v$. Let $E_{u}=E^{\prime} \cap E\left(T_{u}\right)$ and $E_{v}=E^{\prime} \cap E\left(T_{v}\right)$. We notice that properties (i), (ii), and (iii) hold for $E_{u}$ and $E_{v}$ by the same reason as above. Thus, again $T_{u}$ and $T_{v}$ are zig-zag graphs with zig-zag sets $E_{u}$ and $E_{v}$, respectively, and $T$ is obtained from $T_{u}$ and $T_{v}$ by using rule (a). Therefore, $T \in \mathcal{T}$ and the proof is complete.

The Figure 2 represents an example of a tree belonging to the family $\mathcal{T}$.

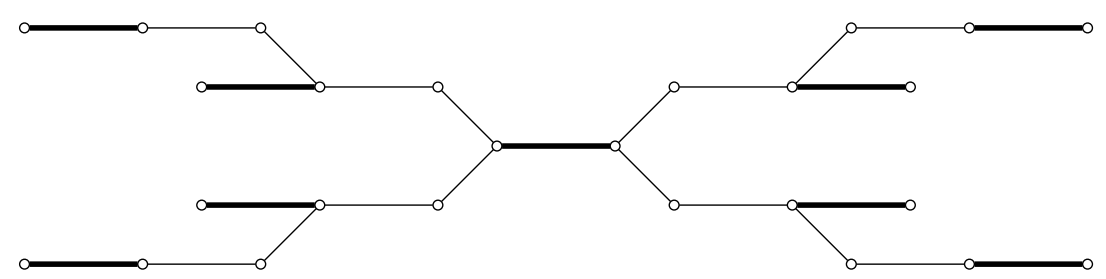

Fig. 2: A tree of the family $\mathcal{T}$ obtained only by rule (a).

\section{Efficient open domination graphs $G \square K_{2}$}

The Cartesian product $G \square H$ of graphs $G$ and $H$ is a graph with $V(G \square H)=V(G) \times V(H)$. Two vertices $(g, h)$ and $\left(g^{\prime}, h^{\prime}\right)$ are adjacent in $G \square H$ whenever $\left(g g^{\prime} \in E(G)\right.$ and $\left.h=h^{\prime}\right)$ or $\left(g=g^{\prime}\right.$ and $\left.h h^{\prime} \in E(H)\right)$. Hence $E(G \otimes H)=E(G \square H) \cup E(G \times H)$. The Cartesian product is commutative and associative (see [10]). Layers and projections are defined identically as for the lexicographic product. The subgraph of $G \square H$ induced by $G^{h}$ or ${ }^{g} H$ is isomorphic to $G$ or $H$, respectively. 
As usual in domination related problems, it seems that the Cartesian product of graphs is the most problematic of all four standard products for the efficient open domination problem. For instance, until now only the Cartesian product of two paths (grid graphs) [4, 14] and some particular case of the Cartesian product of two cycles (torus graphs) [5] have been studied. In this section we characterize all efficient open domination graphs among $G \square K_{2}$.

Cartesian product graphs $G \square K_{2}$ are often called prisms and can be described as two copies of $G$ with a matching between the corresponding vertices of each copy of $G$. We denote $V\left(K_{2}\right)=\{1,2\}$ and for a vertex $v \in V(G)$ we denote by $v^{i}, i \in\{1,2\}$, the copy of $v$ in the $G^{i}$-layer.

With the knowledge about zig-zag graphs we show now the relationship between them and efficient open domination prisms.

Theorem 4.1 If $G$ is a zig-zag graph, then $G \square K_{2}$ is an efficient open domination graph.

Proof. Let $G$ be a zig-zag graph and $E^{\prime}=\left\{e_{1}, \ldots, e_{k}\right\}, e_{i}=u_{i} v_{i}$, its zig-zag set. In addition we may assume that $E^{\prime}$ is ordered such that for every $e_{i}, i>1$, there exists $e_{j}, j<i$, with $d_{G}\left(e_{i}, e_{j}\right)=2$. We call such an edge $e_{j}$ an ancestor of $e_{i}$. We define a subset $D$ of $V\left(G \square K_{2}\right)$ inductively as follows. Let $u_{1}^{1}, v_{1}^{1} \in D$. For an edge $e_{i}, i>1$, with ancestor $e_{j}$ we have either $\left(u_{i}^{1}, v_{i}^{1} \in D\right.$ if $\left.u_{j}^{2}, v_{j}^{2} \in D\right)$ or $\left(u_{i}^{2}, v_{i}^{2} \in D\right.$ if $\left.u_{j}^{1}, v_{j}^{1} \in D\right)$. Since an edge $e_{i}, i>1$, can have many ancestors, we need to show that $D$ is well defined. Suppose not, and let $e_{i}$ be the first edge which has two ancestor $e_{j}$ and $e_{\ell}$ with $u_{j}^{1}, v_{j}^{1} \in D$ and $u_{\ell}^{2}, v_{\ell}^{2} \in D$ (without loss of generality). By the choice of order of $E^{\prime}$ there must exist a sequence $e_{j}, e_{j_{1}}, \ldots, e_{j_{t}}, e_{1}$ with $d\left(e_{j}, e_{j_{1}}\right)=d\left(e_{j_{t}}, e_{1}\right)=d\left(e_{j_{p}}, e_{j_{p+1}}\right)=2$ for $p \in\{1, \ldots, t-1\}$. Notice that every edge of this sequence has index smaller than $i$. Similarly, there exists a sequence $e_{\ell}, e_{\ell_{1}}, \ldots, e_{\ell_{s}}, e_{1}$ with $d\left(e_{\ell}, e_{\ell_{1}}\right)=d\left(e_{\ell_{s}}, e_{1}\right)=d\left(e_{\ell_{p}}, e_{\ell_{p+1}}\right)=2$ for $p \in\{1, \ldots, s-1\}$, where index of every edge is smaller than $i$. Let $e_{k}=e_{j_{k}}=e_{\ell_{p}}$ be the first common edge of these two sequences. For the sequence $e_{j}, e_{j_{1}}, \ldots, e_{j_{k}}, e_{\ell_{p-1}}, e_{\ell_{p-2}}, \ldots, e_{\ell}, e_{i}$ holds that any two consecutive edges are at distance two, and also the first and the last edge are at this distance. By the construction of $D$ and since all indices are smaller than $i$ (with the exception of $e_{i}$ ), we have that consecutive edges lie zig zag in $G \square K_{2}$ : one edge is in $G^{1}$ and the other in $G^{2}$. Hence, from $e_{j}$ to $e_{\ell}$ we have an even number of edges. Together with $e_{i}$ we have an odd number of edges, which contradicts to the property (iv) of the definition of zig-zag sets. Hence, $D$ is well defined and for every pair of edges $e_{i}, e_{j} \in E^{\prime}$ with $d_{G}\left(e_{i}, e_{j}\right)=2$ it follows $u_{i}^{p}, v_{i}^{p}, u_{j}^{q}, v_{j}^{q} \in D$, where $\{p, q\}=\{1,2\}$.

Next we show that $D$ is an efficient open dominating set of $G \square K_{2}$. Notice that if $u_{i}^{j}, v_{i}^{j} \in D, i \in$ $\{1, \ldots, k\}$ and $j \in\{1,2\}$, then they are dominated by $v_{i}^{j}$ and $u_{i}^{j}$, respectively. On the contrary, if $u_{i}^{j}, v_{i}^{j} \notin D$, then $u_{i}^{\ell}$ and $v_{i}^{\ell}$ dominate $u_{i}^{j}$ and $v_{i}^{j}$, respectively, where $\ell \in\{1,2\}-\{j\}$. Let now $w \in$ $V(G)-\left\{u_{i}, v_{i}: i \in\{1, \ldots, k\}\right\}$. By the property (iii) of the definition of zig-zag sets, there exist unique $j$ and $\ell$ with $d\left(w, e_{j}\right)=d\left(w, e_{\ell}\right)=1$. Hence, either $e_{j}$ is an ancestor of $e_{\ell}$ or vice versa. In each case we may assume without loss of generality that $u_{j}^{1}, v_{j}^{1} \in D$ and $u_{\ell}^{2}, v_{\ell}^{2} \in D$. Now $w^{1}$ is dominated by $u_{j}^{1}$ or $v_{j}^{1}$ and $w^{2}$ is dominated by $u_{\ell}^{2}$ or $v_{\ell}^{2}$. Hence $\bigcup_{v \in D} N_{G \square K_{2}}(v)=V\left(G \square K_{2}\right)$. By the property (i) of the definition of zig-zag sets we have that $N_{G \square K_{2}}\left(u_{i}^{j}\right) \cap N_{G \square K_{2}}\left(v_{i}^{j}\right)=\emptyset$ for $u_{i}^{j}, v_{i}^{j} \in D$ and $j \in\{1,2\}$. If $d_{G}\left(e_{i}, e_{j}\right)=2$, then $N_{G \square K_{2}}\left(x_{i}^{p}\right) \cap N_{G \square K_{2}}\left(y_{j}^{q}\right)=\emptyset$ for $x, y \in\{u, v\}$ since $\{p, q\}=\{1,2\}$ by the construction of $D$. Finally, if $d_{G}\left(e_{i}, e_{j}\right)>2$, then clearly $N_{G \square K_{2}}\left(x_{i}^{p}\right) \cap N_{G \square K_{2}}\left(y_{j}^{q}\right)=\emptyset$ for $x, y \in\{u, v\}$ and $p, q \in\{1,2\}$. Therefore, the neighborhoods of $D$ form a partition of $G \square K_{2}$ and, as a consequence, $G \square K_{2}$ is an efficient open domination graph.

Notice that in above proof all edges induced by $D$ project to edges of $G$. However, if we wish that all 
edges induced by an efficient open domination set $D$ of $G \square K_{2}$ projet into $K_{2}$, then it is not hard to see which property is needed for $G$. Namely, if $G$ is 1-perfect graph with 1-perfect code $P$, then $D$ contains exactly $P$ in both copies of $G$. In particular notice that a path graph $P_{k}$ is a 1-perfect graph for every integer $k \geq 1$, and hence $P_{k} \square K_{2}$ is an efficient open domination graph. The following result is clear.

Remark 4.2 If $G$ is a 1-perfect graph, then $G \square K_{2}$ is an efficient open domination graph.

To describe all efficient open domination graphs among Cartesian products of graphs with $K_{2}$ we need a combination of both: 1-perfect graphs and zig-zag graphs. Let $H_{1}$ be 1-perfect graph with 1-perfect code $P$ and let $H_{2}$ be a zig-zag graph with a zig-zag set $E^{\prime}=\left\{e_{1}, \ldots, e_{k}\right\}, e_{i}=u_{i} v_{i}$. (Notice that $H_{1}$ and $H_{2}$ do not need to be connected.) A graph $G$ is called a 1-perfect zig-zag graph if $V(G)=V\left(H_{1}\right) \cup V\left(H_{2}\right)$, $E(G)=E\left(H_{1}\right) \cup E\left(H_{2}\right) \cup E_{3}$ where $E_{3}$ is an arbitrary subset of the set $\left\{x w: x \in V\left(H_{1}\right)-P, w \in\right.$ $\left.V\left(H_{2}\right)-\left\{u_{i}, v_{i}: i \in\{1, \ldots, k\}\right\}\right\}$. In other words, $E(G)$ contains all edges of $H_{1}$, all edges of $H_{2}$ and for every $x \in V\left(H_{1}\right)-P$ and every $w \in V\left(H_{2}\right)-\left\{u_{i}, v_{i}: i \in\{1, \ldots, k\}\right\}$ we may insert an edge $x w$ to $E(G)$ or not. In particular, $G$ is isomorphic to the disjoint union of $H_{1}$ and $H_{2}$ if no edges of the type $x w$ are added.

Notice that a 1-perfect zig-zag graph could be formed only by a 1-perfect graph or only by a zig-zag graph. This means that, for instance, all paths (which are always 1-perfect graphs, but not always zig-zag graphs) are included into the family of 1-perfect zig-zag graphs. While it is easy to see that there exists 1-perfect graphs which are not zig-zag graphs, one needs a bit more effort to find a graph that is a zig-zag, but not a 1-perfect graph. An example for this is $K_{2 n}^{+}$. Recall that we obtain $K_{2 n}^{+}$by subdividing with one vertex every edge which does not belong to a fixed perfect matching of $K_{2 n}$.

Theorem 4.3 Let $G$ be a graph. The Cartesian product $G \square K_{2}$ is an efficient open domination graph if and only if $G$ is a 1-perfect zig-zag graph.

Proof. First, if $G$ is formed either by a zig-zag graph or by a 1-perfect graph, then by Theorem 4.1 or by Remark 4.2, respectively, we obtain that $G \square K_{2}$ is an efficient open domination graph.

Now, let $G$ be a 1-perfect zig-zag graph built from 1-perfect graph $H_{1}$ with 1-perfect code $P$ and zigzag graph $H_{2}$ with zig-zag set $E^{\prime}=\left\{e_{1}, \ldots, e_{k}\right\}, e_{i}=u_{i} v_{i}$. Let $P^{i}, i \in\{1,2\}$, be a copy of $P$ in the $G^{i}$-layer of $G \square K_{2}$ and let $D$ be a set of vertices obtained from $E^{\prime}$ as in the proof of Theorem 4.1. The set $D^{\prime}=P^{1} \cup P^{2} \cup D$ dominates $G \square K_{2}$ since $P^{1} \cup P^{2}$ dominates $H_{1} \square K_{2}$ and $D$ dominates $H_{2} \square K_{2}$. In addition, $N_{G \square K_{2}}(x) \cap N_{G \square K_{2}}(y)=\emptyset$ for any $x, y \in D^{\prime}$. Finally, since all additional edges in $G$ between $H_{1}$ and $H_{2}$ are connecting vertices which neither belong to $P$ nor to $\left\{u_{i}, v_{i}: i \in\{1, \ldots, k\}\right\}$, we obtain that $G \square K_{2}$ is an efficient open domination graph.

For the other direction, let $G \square K_{2}$ be an efficient open domination graph with an efficient open dominating set $D$. Clearly $D$ contains adjacent pairs of vertices and we split them into two subsets as follows. In $D_{1}$ we put pairs of adjacent vertices from $D$ for which its edge projects to the edge of $K_{2}$ and in $D_{2}$ are all the remaining vertices (those whose edge projects to an edge of $G$ ). First we define a graph $H_{1}$ as follows. Let $V\left(H_{1} \square K_{2}\right)=\bigcup_{v \in D_{1}} N_{G \square K_{2}}(v)$ and $H_{1} \square K_{2}$ is an induced subgraph of $G \square K_{2}$ on $V\left(H_{1} \square K_{2}\right)$. It is clear that $H_{1}$ which is induced by vertices of layer $H_{1}^{1}$ (and also by vertices of layer $\left.H_{1}^{2}\right)$ is a 1-perfect graph with 1-perfect code $p_{G}\left(D_{1}\right)$.

Next, let $H_{2} \square K_{2}=G \square K_{2}-V\left(H_{1} \square K_{2}\right)$. The projection of all edges induced by $D_{2}$ projects to edges of the first factor $H_{2}$. We will show that these edges, namely $p_{H_{2}}\left(D_{2}\right)$, form a zig-zag set of $H_{2}$. Let $\left|D_{2}\right|=2 k$ and denote adjacent vertices of $D_{2}$ by $u_{i}^{j}$ and $v_{i}^{j}$ for $i \in\{1, \ldots, k\}$ and $j \in\{1,2\}$. Since $D_{2}$ 
is a subset of an efficient open dominating set $D$, it follows $N_{H_{2} \square K_{2}}\left(u_{i}^{j}\right) \cap N_{H_{2} \square K_{2}}\left(v_{i}^{j}\right)=\emptyset$ for every $i \in\{1, \ldots, k\}$ and $j \in\{1,2\}$, consequently $N_{H_{2}}\left(u_{i}\right) \cap N_{H_{2}}\left(v_{i}\right)=\emptyset$ and the property (i) holds.

For the property (ii), let $e_{1}=u_{1} v_{1}$ and $e_{2}=u_{2} v_{2}$ be two different edges induced by $p_{H_{2}}\left(D_{2}\right)$ and let $e_{1}^{\prime}, e_{2}^{\prime} \in E\left(H_{2} \square K_{2}\right)$ be edges which project to $e_{1}$ and $e_{2}$, respectively. If $d_{H_{2}}\left(e_{1}, e_{2}\right)=0$, then two end vertices coincide and the other two differ. Without loss of generality, let $u_{1}=u_{2}$ and $v_{1} \neq v_{2}$. If both $e_{1}^{\prime}$ and $e_{2}^{\prime}$ lie in the same $H_{2}$-layer, say $H_{2}^{1}$, then $u_{1}^{1} \in N_{H_{2} \square K_{2}}\left(v_{1}^{1}\right) \cap N_{H_{2} \square K_{2}}\left(v_{2}^{1}\right)$ which is not possible, since $D$ is an efficient open dominating set. If $e_{1}^{\prime}$ and $e_{2}^{\prime}$ lie in different $H_{2}$-layers, say $e_{1}^{\prime}$ in $H_{2}^{1}$ and $e_{2}^{\prime}$ in $H_{2}^{2}$, then $u_{1}^{1} \in N_{H_{2} \square K_{2}}\left(v_{1}^{1}\right) \cap N_{H_{2} \square K_{2}}\left(u_{2}^{1}\right)$, which again yields to the same contradiction. If $d_{H_{2}}\left(e_{1}, e_{2}\right)=1$, then there are two end vertices, say $u_{1}$ and $u_{2}$, adjacent in $H_{2}$. If both $e_{1}^{\prime}$ and $e_{2}^{\prime}$ lie in the same $H_{2}$-layer, say $H_{2}^{1}$, then $u_{1}^{1} \in N_{H_{2} \square K_{2}}\left(v_{1}^{1}\right) \cap N_{H_{2} \square K_{2}}\left(u_{2}^{1}\right)$, which is not possible by the same reason. If $e_{1}^{\prime}$ and $e_{2}^{\prime}$ lie in different $H_{2}$-layers, say $e_{1}^{\prime}$ in $H_{2}^{1}$ and $e_{2}^{\prime}$ in $H_{2}^{2}$, then $u_{1}^{2} \in N_{H_{2} \square K_{2}}\left(u_{1}^{1}\right) \cap N_{H_{2} \square K_{2}}\left(u_{2}^{2}\right)$, which is not possible. Hence, $d_{H_{2}}\left(e_{1}, e_{2}\right) \geq 2$ and the property (ii) holds for $p_{H_{2}}\left(D_{2}\right)$.

Let $w \in V\left(H_{2}\right)-p_{H_{2}}\left(D_{2}\right)$. Hence both $w^{1}$ and $w^{2}$ are not in $D_{2}$. Suppose that they are dominated by $u^{1}$ and $u^{2}$, respectively. Both $u^{1}$ and $u^{2}$ have a neighbor in $D_{2}$ in the same layer: $v^{1}$ and $v^{2}$, respectively. Let $e=u^{1} v^{1}$ and $e^{\prime}=u^{2} v^{2}$. If $p_{H_{2}}(e)=p_{H_{2}}\left(e^{\prime}\right)$, then $u^{1} \in N_{H_{2} \square K_{2}}\left(v^{1}\right) \cap N_{H_{2} \square K_{2}}\left(u^{2}\right)$, which is not possible. Moreover, by the property (ii) we have $d_{H_{2}}\left(p_{H_{2}}(e), p_{H_{2}}\left(e^{\prime}\right)\right)=2$. Clearly $d_{H_{2}}\left(w, p_{H_{2}}(e)\right)=$ $d_{H_{2}}\left(w, p_{H_{2}}\left(e^{\prime}\right)\right)=1$. If there exists a third edge $p_{H_{2}}\left(e^{\prime \prime}\right)$ induced by $p_{H_{2}}\left(D_{2}\right)$ with $d_{H_{2}}\left(w, p_{H_{2}}\left(e^{\prime \prime}\right)\right)=$ 1 , then either $w^{1}$ or $w^{2}$ is dominated by two vertices of $D_{2} \subseteq D$, which is not possible, and the property (iii) is satisfied by $p_{H_{2}}\left(D_{2}\right)$.

If the property (iv) does not hold, then there exists a sequence of distinct edges $e_{i_{1}}, e_{i_{2}}, \ldots, e_{i_{j}}, j>2$, with $d\left(e_{i_{\ell}}, e_{i_{\ell+1(\bmod j)}}\right)=2$ for $\ell \in\{1, \ldots, j\}$ and $j$ is an odd number. Since $d\left(e_{i_{\ell}}, e_{i_{\ell+1(\bmod j)}}\right)=2$ for $\ell \in\{1, \ldots, j\}$, there exists a vertex $w_{\ell}$ for which $d\left(w_{\ell}, e_{i_{\ell}}\right)=1=d\left(w_{\ell}, e_{i_{\ell+1(\bmod j)}}\right)$. By $e_{i_{\ell}}^{\prime}$ we denote the edge which projects to $e_{i_{j}}$ for every $\ell \in\{1, \ldots, j\}$. Two consecutive edges $e_{i_{p}}^{\prime}$ and $e_{i_{p+1(\bmod j)}}^{\prime}$ must be in the same $H_{2}$-layer, say $H_{2}^{1}$, since $j$ is odd. Without loss of generality, we may assume that $w_{p}^{1}$ is a common neighbor of $u_{i_{p}}^{1}$ and $v_{i_{p+1(\bmod j)}}^{1}$. Thus $w_{p}^{1} \in N_{H_{2} \square K_{2}}\left(u_{i_{p}}^{1}\right) \cap N_{H_{2} \square K_{2}}\left(v_{i_{p+1(\bmod j)}}^{1}\right)$, a final contradiction. Hence, the property (iv) also holds, $p_{H_{2}}\left(D_{2}\right)$ is a zig-zag set of $H_{2}$ and $H_{2}$ is a zig-zag graph. Therefore, $G$ is 1-perfect zig-zag graph, which ends the proof.

To generalize this results from $K_{2}$ to $K_{p}, p>2$, it is easy to see that no edge induced by an efficient open dominating set $D$ of $G \square K_{p}$ can project to $K_{p}$. Hence 1-perfect graphs have no analogue for $p>2$. However, it seems that zig-zag graphs could be generalized to higher orders of $K_{p}$, where property (iv) represents the greatest problem.

\section{Grid-like graphs}

The study of efficient open domination graphs among Cartesian product of path and/or cycles has attracted some researchers in the last few years. For instance, [4, 14] are dedicated to investigate the efficient open domination graphs among the grid graphs $P_{r} \square P_{t}$. Between both works all the efficient open domination grid graphs were characterized. Notice that, in [4], efficient open dominating sets are referred as exact transversals, while in [14], are called total perfect codes. The union of these two works produced the following characterization.

Theorem 5.1 [4, 14] A grid graph $P_{r} \square P_{t}, t \geq r \geq 3$, is an efficient open domination graph if and only if $r$ is an even number and $t \equiv x(\bmod \mathrm{r}+1)$ for $x \in\{1, r-2, r\}$. 
Some partial results on the efficient open domination graphs among torus graphs $C_{r} \square C_{t}$ were presented in [5]. There was given the following characterization of all the torus graphs $C_{r} \square C_{t}$ having an efficient open dominating set where all its edges are parallel (a parallel total perfect code).

Theorem 5.2 [5] A parallel total perfect code in a Cartesian product graph $C_{r} \square C_{t}$ of two cycles $C_{r}$ and $C_{t}$ exists if and only if $r$ and $t$ are multiples of four.

From now on we give some partial results on the torus $C_{r} \square C_{t}$ and the cylinder $P_{r} \square C_{t}$. To do so, in this subsection we use the following notation: $U=\left\{u_{0}, \ldots, u_{r-1}\right\}$ and $V=\left\{v_{0}, \ldots, v_{t-1}\right\}$ are the vertex sets of $G$ and $H$, where $G$ and $H$ are isomorphic to a path or a cycle of order $r$ and $t$, respectively. Operations with the subindexes of vertices of $U$ and $V$ are done modulo $r$ and $t$, respectively. With respect to the previous section we may assume that $r, t \geq 3$. The adjacency in $G$ and $H$ is defined as $u_{0} \sim u_{1} \sim \ldots \sim u_{r-1}\left(\sim u_{0}\right)$ and $v_{0} \sim v_{1} \sim \ldots \sim v_{t-1}\left(\sim v_{0}\right)$, respectively.

\subsection{The torus $C_{r} \square C_{t}$}

By Theorem 5.2 we know that $C_{4 r} \square C_{4 t}$ are efficient open domination graphs. Motivated by this we state the following conjecture.

Conjecture 5.3 The torus $C_{r} \square C_{t}, r, t \geq 3$, is an efficient open domination graph if and only if $r, t \equiv$ $0(\bmod 4)$.

Till the end of this subsection we settle this conjecture for $r \in\{3,4,5,6,7\}$. We start with $r=4$ and continue with all other values.

Proposition 5.4 Torus $C_{4} \square C_{t}, t \geq 4$, is an efficient open domination graph if and only ift $\equiv 0(\bmod 4)$.

Proof. If $t \equiv 0(\bmod 4)$, then it follows by Theorem 5.2 that $C_{4} \square C_{t}$ is an efficient open domination graph.

Now suppose that the torus graph $C_{4} \square C_{t}, t \geq 4$, is an efficient open domination graph and let $F$ be an efficient open dominating set in $C_{4} \square C_{t}$. By Observation 1.2 we have that $t$ is even. So either $t \equiv 0(\bmod 4)$ or $t \equiv 2(\bmod 4)$. Suppose $t \equiv 2(\bmod 4)$. According to the symmetry of $C_{4} \square C_{t}$, we can suppose, without loss of generality, that $\left(u_{0}, v_{0}\right) \in F$ and $\left(u_{0}, v_{t-1}\right) \notin F$.

If $\left(u_{0}, v_{1}\right) \in F$, then we have that $\left(u_{2}, v_{2}\right),\left(u_{2}, v_{3}\right) \in F$ and consequently, the vertices $\left(u_{0}, v_{4}\right)$, $\left(u_{0}, v_{5}\right),\left(u_{2}, v_{6}\right),\left(u_{2}, v_{7}\right), \ldots,\left(u_{0}, v_{t-2}\right),\left(u_{0}, v_{t-1}\right)$ also belong to $F$, which is a contradiction since $\left(u_{0}, v_{t-1}\right)$ is dominated by $\left(u_{0}, v_{0}\right)$ and $\left(u_{0}, v_{t-2}\right)$. Analogously, we obtain a contradiction if $\left(u_{1}, v_{0}\right) \in$ $F$. Therefore $t \equiv 0(\bmod 4)$.

Theorem 5.5 Torus $C_{r} \square C_{t}, r \in\{3,5,6,7\}$ and $t \geq r$, is not an efficient open domination graph.

Proof. If $r=3$, then it is straightforward to observe that $C_{3} \square C_{t}$ is not an efficient open domination graph for every $t \geq 3$. Now suppose $r=5$ and let $F_{5}$ be an efficient open dominating set in $C_{5} \square C_{t}$. According to the symmetry of $C_{5} \square C_{t}$, we consider without loss of generality that $\left(u_{0}, v_{0}\right) \in F_{5}$ and $\left(u_{0}, v_{t-1}\right) \notin F_{5}$. If $\left(u_{1}, v_{0}\right) \in F_{5}$, then we have that $\left(u_{3}, v_{1}\right),\left(u_{3}, v_{2}\right) \in F_{5}$ and consequently, $\left(u_{0}, v_{3}\right),\left(u_{1}, v_{3}\right) \in F_{5}$. Thus, we have that at least one of the vertices of the set $\left\{\left(u_{2}, v_{4}\right),\left(u_{3}, v_{4}\right),\left(u_{4}, v_{4}\right)\right\}$ cannot be efficiently open dominated by $F_{5}$, a contradiction. On the other hand, if $\left(u_{0}, v_{1}\right) \in F_{5}$, then $\left(u_{2}, v_{2}\right),\left(u_{3}, v_{2}\right) \in F_{5}$ and at least one of the vertices of the set $\left\{\left(u_{0}, v_{3}\right),\left(u_{1}, v_{3}\right),\left(u_{4}, v_{3}\right)\right\}$ cannot be efficiently open dominated by $F_{5}$, a contradiction again. 
Suppose $r=6$ and let $F_{6}$ be an efficient open dominating set in $C_{6} \square C_{t}$. We proceed similarly to the above case. We may assume that $\left(u_{0}, v_{0}\right) \in F_{6}$ and $\left(u_{0}, v_{t-1}\right) \notin F_{6}$. If $\left(u_{0}, v_{1}\right) \in F_{6}$, then we have the following cases.

Case 1: $\left(u_{3}, v_{0}\right),\left(u_{3}, v_{1}\right) \in F_{6}$. As a consequence, we have that two vertices of the set $\left\{\left(u_{1}, v_{2}\right),\left(u_{2}, v_{2}\right)\right.$, $\left.\left(u_{4}, v_{2}\right),\left(u_{5}, v_{2}\right)\right\}$ cannot be efficiently open dominated by $F_{6}$, a contradiction.

Case 2: $\left(u_{3}, v_{1}\right),\left(u_{3}, v_{2}\right) \in F_{6}$. Consequently, either $\left(u_{2}, v_{0}\right)$ or $\left(u_{4}, v_{0}\right)$ cannot be efficiently open dominated by $F_{6}$, a contradiction.

Case 3: Either $\left(u_{2}, v_{2}\right),\left(u_{2}, v_{3}\right) \in F_{6}$ or $\left(u_{4}, v_{2}\right),\left(u_{4}, v_{3}\right) \in F_{6}$. Consequently, either $\left(u_{4}, v_{1}\right)$ or $\left(u_{2}, v_{1}\right)$, respectively, cannot be efficiently open dominated by $F_{6}$, a contradiction.

Case 4: $\left(u_{3}, v_{2}\right),\left(u_{3}, v_{3}\right) \in F_{6}$. Consequently, $\left(u_{2}, v_{1}\right)$ and $\left(u_{4}, v_{1}\right)$ cannot be efficiently open dominated by $F_{6}$, a contradiction.

Case 5: Either $\left(u_{2}, v_{2}\right),\left(u_{3}, v_{2}\right) \in F_{6}$ or $\left(u_{3}, v_{2}\right),\left(u_{4}, v_{2}\right) \in F_{6}$. Consequently, either $\left(u_{4}, v_{1}\right)$ or $\left(u_{2}, v_{1}\right)$, respectively, cannot be efficiently open dominated by $F_{6}$, a contradiction.

On the other hand, if $\left(u_{1}, v_{0}\right) \in F_{6}$, then we have the following cases.

Case 6: $\left(u_{3}, v_{1}\right),\left(u_{4}, v_{1}\right) \in F_{6}$. As a consequence, we have that two vertices of the set $\left\{\left(u_{0}, v_{2}\right),\left(u_{1}, v_{2}\right)\right.$, $\left.\left(u_{2}, v_{2}\right)\left(u_{5}, v_{2}\right)\right\}$ cannot be efficiently open dominated by $F_{6}$, a contradiction.

Case 7: Either $\left(u_{3}, v_{1}\right),\left(u_{3}, v_{2}\right) \in F_{6}$ or $\left(u_{4}, v_{1}\right),\left(u_{4}, v_{2}\right) \in F_{6}$. Consequently, either $\left(u_{5}, v_{1}\right)$ or $\left(u_{2}, v_{1}\right)$, respectively, cannot be efficiently open dominated by $F_{6}$, a contradiction.

Now, if $r=7$, then by using a similar cases analysis like in the constructive procedure of the set $F_{6}$ for $r=6$, we obtain contradictions which lead to that $C_{7} \square C_{t}$ is not an efficient open domination graph. We leave the details to the reader.

\subsection{The cylinder $P_{r} \square C_{t}$}

As we will see, the situation for cylinders is a little bit more complicated. The first indicator for this is that $P_{2} \square C_{t} \cong C_{t} \square K_{2}$ and, by Theorem 4.3 , we know that this is an efficient open domination graph whenever $t \equiv 0(\bmod 3)$. Also other cases are more complicated. We start with cycles on $4 t$ vertices.

Proposition 5.6 Cylinder $P_{2 r+1} \square C_{4 t}$ is an efficient open domination graph for every $r, t \geq 1$.

Proof. The result follows immediately from the fact that the set $F$ of vertices of $P_{2 r+1} \square C_{4 t}$ given in the following way is an efficient open dominating set for $P_{2 r+1} \square C_{4 t}$.

If $2 r+1 \equiv 1(\bmod 4)$, then the set $F$ is the union of sets $\left\{u_{0}, u_{4}, \ldots, u_{2 r}\right\} \times\left\{v_{0}, v_{1}, v_{4}, v_{5}, \ldots\right.$, $\left.v_{4 t-4}, v_{4 t-3}\right\}$ and $\left\{u_{2}, u_{6}, \ldots, u_{2 r-2}\right\} \times\left\{v_{2}, v_{3}, v_{6}, v_{7}, \ldots, v_{4 t-2}, v_{4 t-1}\right\}$.

If $2 r+1 \equiv 3(\bmod 4)$, then the set $F$ is the union of sets $\left\{u_{0}, u_{4}, \ldots, u_{2 r-2}\right\} \times\left\{v_{0}, v_{1}, v_{4}, v_{5}, \ldots\right.$, $\left.v_{4 t-4}, v_{4 t-3}\right\}$ and $\left\{u_{2}, u_{6}, \ldots, u_{2 r}\right\} \times\left\{v_{2}, v_{3}, v_{6}, v_{7}, \ldots, v_{4 t-2}, v_{4 t-1}\right\}$.

Next we see characterizations for $P_{r} \square C_{t}$ to be efficient open domination graphs for $t \in\{3,4,5,6,7\}$.

\section{Theorem 5.7}

(i) $P_{r} \square C_{3}$ is an efficient open domination graph if and only if $r=2$.

(ii) $P_{r} \square C_{4}$ is an efficient open domination graph if and only if $r$ is an odd integer.

(iii) $P_{r} \square C_{5}$ is an efficient open domination graph if and only if $r=4$. 
(iv) $P_{r} \square C_{6}$ is an efficient open domination graph if and only if $r=2$.

(v) $P_{r} \square C_{7}$ is an efficient open domination graph if and only if $r=6$.

Proof. It is easy to observe that $P_{2} \square C_{3}, P_{4} \square C_{5}, P_{2} \square C_{6}$ and $P_{6} \square C_{7}$ are efficient open domination graphs, while $P_{2 t+1} \square C_{4}$ is efficient open domination graph by Proposition 5.6. Also, it is straightforward to check that if $P_{r} \square C_{3}$ is an efficient open domination graph, then $r=2$. So (i) is proved.

For (ii) suppose that cylinder $P_{r} \square C_{4}, r \geq 4$, is an efficient open domination graph and let $F$ be an efficient open dominating set in $P_{r} \square C_{4}$. Clearly, at least one vertex of ${ }^{u_{0}} C_{4}$ must be in $F$ and according to the symmetry of $P_{r} \square C_{4}$, we can suppose, without loss of generality, that $\left(u_{0}, v_{0}\right) \in F$. If $\left(u_{1}, v_{0}\right) \in F$, then we have that the vertex $\left(u_{0}, v_{2}\right)$ cannot be efficiently open dominated by $F$, a contradiction. Now, if $\left(u_{0}, v_{1}\right) \in F$, then $\left(u_{2}, v_{2}\right),\left(u_{2}, v_{3}\right) \in F$, and consequently only vertices of the type $\left(u_{2 k}, v_{2}\right),\left(u_{2 k}, v_{3}\right),\left(u_{4 \ell}, v_{0}\right),\left(u_{4 \ell}, v_{1}\right)$ belong to $F$, where $k, \ell$ are integers and $k$ is odd. Thus, if $r$ is even, then there exist two vertices $\left(u_{r-1}, v_{i}\right),\left(u_{r-1}, v_{j}\right), i \neq j$, and $i, j \in\{0,1,2,3\}$, which cannot be efficiently open dominated by $F$, a contradiction. Therefore, $r$ is odd and (ii) is clear.

Now, suppose $P_{r} \square C_{5}$ is an efficient open domination graph and let $F_{5}$ be an efficient open dominating set in $P_{r} \square C_{5}$. Clearly, at least one vertex of ${ }^{u_{0}} C_{5}$ must be in $F_{5}$ and according to the symmetry of $P_{r} \square C_{5}$, we consider without loss of generality that $\left(u_{0}, v_{0}\right) \in F_{5}$ and $\left(u_{0}, v_{4}\right) \notin F_{5}$. If $\left(u_{1}, v_{0}\right) \in F_{5}$, then we vertices $\left(u_{0}, v_{2}\right)$ and $\left(u_{0}, v_{3}\right)$ cannot be efficiently open dominated by $F_{5}$, a contradiction. On the other hand, let $\left(u_{0}, v_{1}\right) \in F_{5}$. If $r=2$, then $\left(u_{0}, v_{3}\right)$ is not efficiently dominated. If $r>2$, then $\left(u_{1}, v_{3}\right),\left(u_{2}, v_{3}\right) \in F_{5}$. If $r=3$, then $\left(u_{2}, v_{0}\right)$ and $\left(u_{2}, v_{1}\right)$ are not efficiently open dominated by $F_{5}$. Hence $r>3$ and also $\left(u_{3}, v_{0}\right),\left(u_{3}, v_{1}\right) \in F_{5}$. Now, if $r>4$, then one vertex of the set $\left\{\left(u_{4}, v_{2}\right),\left(u_{4}, v_{3}\right),\left(u_{4}, v_{4}\right)\right\}$ cannot be efficiently open dominated by $F_{5}$, a contradiction again. Thus, the only possible choice is $r=4$ and (ii) is proved.

Assume $P_{r} \square C_{6}$ is an efficient open domination graph and let $F_{6}$ be an efficient open dominating set in $P_{r} \square C_{6}$. We proceed similarly to the above case. Consider $\left(u_{0}, v_{0}\right) \in F_{6}$ and $\left(u_{0}, v_{5}\right) \notin F_{6}$. If $\left(u_{1}, v_{0}\right) \in F_{6}$, then $\left(u_{0}, v_{3}\right),\left(u_{1}, v_{3}\right) \in F_{6}$. If $r>2$, then two vertices of the set $\left\{\left(u_{2}, v_{1}\right),\left(u_{2}, v_{2}\right)\right.$, $\left.\left(u_{2}, v_{4}\right),\left(u_{2}, v_{5}\right)\right\}$ cannot be efficiently open dominated by $F_{6}$, a contradiction. On the other hand, if $\left(u_{0}, v_{1}\right) \in F_{6}$, then $\left(u_{1}, v_{3}\right),\left(u_{1}, v_{4}\right) \in F_{6}$. Analogously, if $r>2$, then two vertices of the set $\left\{\left(u_{2}, v_{0}\right),\left(u_{2}, v_{1}\right),\left(u_{2}, v_{2}\right)\right.$,

$\left.\left(u_{2}, v_{5}\right)\right\}$ cannot be efficiently open dominated by $F_{6}$, a contradiction. So, the only possible choice is $r=2$ and (iii) is proved.

Finally, suppose $P_{r} \square C_{7}$ is an efficient open domination graph and let $F_{7}$ be an efficient open dominating set in $P_{r} \square C_{7}$. As above we consider $\left(u_{0}, v_{0}\right) \in F_{7}$ and $\left(u_{0}, v_{6}\right) \notin F_{7}$. If $\left(u_{1}, v_{0}\right) \in F_{7}$, then $\left(u_{0}, v_{2}\right)$ and $\left(u_{0}, v_{5}\right)$ can be efficiently open dominated only by $\left(u_{0}, v_{3}\right)$ and $\left(u_{0}, v_{4}\right)$, respectively. Also $r>2$, since $\left(u_{1}, v_{2}\right)$ and $\left(u_{1}, v_{5}\right)$ are not efficiently open dominated yet. Now $\left(u_{1}, v_{2}\right)$ and $\left(u_{1}, v_{5}\right)$ can be efficiently open dominated only by $\left(u_{2}, v_{2}\right)$ and $\left(u_{2}, v_{5}\right)$, respectively, and these two with $\left(u_{3}, v_{2}\right)$ and $\left(u_{3}, v_{5}\right)$, respectively. Hence $r>3$ and $r \neq 4$ since $\left(u_{3}, v_{0}\right)$ is not efficiently dominated. The vertex $\left(u_{3}, v_{0}\right)$ yields that $\left(u_{4}, v_{0}\right),\left(u_{5}, v_{0}\right) \in F_{7}$ and consequently $r \geq 6$. To continue, $\left(u_{5}, v_{3}\right),\left(u_{5}, v_{4}\right) \in F_{7}$ to efficiently open dominate $\left(u_{4}, v_{3}\right)$ and $\left(u_{4}, v_{4}\right)$. If $r=6$, then we are done. If $r>6$, then at least one vertex out of $\left\{\left(u_{6}, v_{1}\right),\left(u_{6}, v_{2}\right),\left(u_{6}, v_{5}\right),\left(u_{6}, v_{6}\right)\right\}$ cannot be efficiently open dominated by $F_{7}$, a contradiction. On the other hand, if $\left(u_{0}, v_{1}\right) \in F_{7}$, then $\left(u_{0}, v_{4}\right),\left(u_{1}, v_{4}\right) \in F_{7}$. If $r=2$, then $\left(u_{1}, v_{2}\right)$ and $\left(u_{1}, v_{6}\right)$ are not dominated by $F_{7}$, a contradiction. Hence $r>2$ and to efficiently open dominate these two vertices, $\left(u_{2}, v_{2}\right)$ and $\left(u_{2}, v_{6}\right)$, respectively, must be in $F_{7}$. Furthermore, to efficiently open dominate $\left(u_{2}, v_{2}\right)$ and $\left(u_{2}, v_{6}\right),\left(u_{3}, v_{2}\right)$ and $\left(u_{3}, v_{6}\right)$, respectively, must be in $F_{7}$. Hence $r>3$ and if 
$r=4$, then $\left(u_{3}, v_{4}\right)$ is not dominated by $F_{7}$. Thus, $r>4$ and to efficiently open dominate $\left(u_{3}, v_{4}\right)$ we need $\left(u_{4}, v_{4}\right),\left(u_{5}, v_{4}\right) \in F_{7}$. Moreover, the vertices $\left(u_{5}, v_{0}\right),\left(u_{5}, v_{1}\right) \in F_{7}$ to efficiently open dominate $\left(u_{4}, v_{0}\right)$ and $\left(u_{4}, v_{1}\right)$. If $r=6$, then we are done and if $r>6$, then at least one vertex of the set $\left\{\left(u_{6}, v_{2}\right),\left(u_{6}, v_{3}\right),\left(u_{6}, v_{5}\right),\left(u_{6}, v_{6}\right)\right\}$ cannot be efficiently open dominated by $F_{7}$, a contradiction. Therefore, the only possible choice is $r=6$ and (iv) is proved.

\section{References}

[1] G. Abay-Asmerom, R. H. Hammack, D. T. Taylor, Total perfect codes in tensor products of graphs. Ars Combin., 88:129-134, 2008.

[2] B. Brešar, P. Dorbec, W. Goddard, B. Hartnell, M. A. Henning, S. Klavžar, and D. F. Rall. Vizing's conjecture: a survey and recent results. J. Graph Theory, 69:46-76, 2012.

[3] E.J. Cockayne, B.L. Hartnell, S.T. Hedetniemi, and R. Laskar, Perfect domination in graphs, J. Comb. Info. System Sci., 18:136-148, 1993.

[4] R. Cowen, S. H. Hechler, J. W. Kennedy, and A. Steinberg, Odd neighborhood transversals on grid graphs, Discrete Math., 307:2200-2208, 2007.

[5] I. J. Dejter, Perfect domination in regular grid graphs, Australas. J. Combin., 42:99-114, 2008.

[6] P. Dorbec, S. Gravier, S. Klavžar, and S. Špacapan. Some results on total domination in direct products of graphs. Discuss. Math. Graph Theory, 26:103-112, 2006.

[7] H. Gavlas and K. Schultz. Efficient open domination. Electron. Notes Discrete Math., 11:681-691, 2002.

[8] H. Gavlas, K. Schultz, and P. Slater. Efficient open domination in graphs. Sci. Ser. A Math. Sci., 6:77-84, 2003.

[9] S. Gravier. Total domination number of grid graphs. Discrete Appl. Math., 121:119-128, 2002.

[10] R. Hammack, W. Imrich, and S. Klavžar, Handbook of Product Graphs, Second Edition. CRC Press, Boca Raton, FL, 2011.

[11] J. Jerebic, S. Klavžar, and S. Špacapan. Characterizing $r$-perfect codes in direct products of two and three cycles. Inf. Process. Lett., 94:1-6, 2005.

[12] S. Klavžar, S. Špacapan, and J. Žerovnik. An almost complete description of perfect codes in direct products of cycles. Adv. Appl. Math., 37:2-18, 2006.

[13] A. Klobučar. Total domination number of cartesian products. Math. Commun., 9:35-44, 2004.

[14] W.F. Klostermeyer and J.L. Goldwasser, Total Perfect Codes in Grid Graphs, Bull. Inst. Combin. Appl., 46:61-68, 2006. 
[15] D. Kuziak, I. Peterin, and I. G. Yero. Erratum to "Total domination number of cartesian products" [Math. Commun. 9 (2004) 35-44]. Math. Commun., in press.

[16] A. McRae, Generalizing NP-completeness Proofs for Bipartite Graphs and Chordal Graphs, PhD Thesis, Clemson University, 1994

[17] G. Mekiš. Lower bounds for the domination number and the total domination number of direct product graphs. Discrete Math., 310:3310-3317, 2010.

[18] O. Ore, Theory of Graphs, Colloquium Publications, Volume 38, American Mathematical Society, 1962.

[19] E. R. Scheinerman and D. H. Ullman, Fractional Graph Theory, Series in Discrete Mathematics and Optimization, Wiley-Interscience, 1997.

[20] T. Tamizh Chelvam. Efficient open domination in Cayley graphs. Appl. Math. Lett., 25:1560-1564, 2012. 